\title{
Activation of formyl peptide receptor 2 by WKYMVm enhances emergency granulopoiesis through phospholipase $\mathrm{C}$ activity
}

\author{
Hyung Sik Kim ${ }^{1}$, Min Young Park ${ }^{1}$, Sung Kyun Lee ${ }^{1,4}$, Joon Seong Park ${ }^{2}$, Ha Young Lee ${ }^{1}$ E Yoe-Sik Bae $e^{1,3, *}$ \\ ${ }^{1}$ Department of Biological Sciences, Sungkyunkwan University, Suwon 16419, ${ }^{2}$ Department of Hematology-Oncology, Ajou University \\ School of Medicine, Suwon 16499, ${ }^{3}$ Department of Health Sciences and Technology, SAIHST, Sungkyunkwan University, Seoul 06351, \\ Korea, ${ }^{4}$ Present address: Institute for Stem Cell \& Regenerative Medicine Research of Albert Einstein College of Medicine, Bronx, NY \\ 10461, USA
}

\begin{abstract}
Emergency granulopoiesis is a very important strategy to supply efficient neutrophil number in response to infection. However, molecular mechanism involved in this process remains unclear. Here, we found that administration of WKYMVm, an immune modulating peptide, to septic mice strongly increased neutrophil number through augmented emergency granulopoiesis. WKYMVm-induced emergency granulopoiesis was blocked not only by a formyl peptide receptor 2 (FPR2) antagonist (WRW4), but also by FPR2 deficiency. As progenitors of neutrophils, Lin $^{-} \mathrm{C}-\mathrm{kit}^{+} \mathrm{Sca}-\mathbf{1}^{-}$ cells expressed FPR2. WKYMVm-induced emergency granulopoiesis was also blocked by a phospholipase $\mathrm{C}$ inhibitor (U-73122). These results suggest that WKYMVm can stimulate emergency granulopoiesis via FPR2 and phospholipase C enzymatic activity. [BMB Reports 2018; 51(8): 418-423]
\end{abstract}

\section{INTRODUCTION}

Neutrophils play an essential role in host defense against bacterial infection $(1,2)$. Neutrophils can engulf invading pathogens and remove them via several different mechanisms $(1,2)$. Neutrophils generate reactive oxygen species (ROS) through activation of NADPH oxidase and these ROS can kill invading pathogens (3). Since neutrophils play important innate immune responses against pathogen infection $(1,2)$, regulation of de novo neutrophil generation is a hot issue.

*Corresponding author. Tel: +82-31-290-5914; Fax: +82-31-2907015; E-mail: yoesik@skku.edu

https://doi.org/10.5483/BMBRep.2018.51.8.080

Received 13 April 2018, Revised 4 June 2018, Accepted 3 July 2018

Keywords: Emergency granulopoiesis, Formyl peptide receptor 2, Phospholipase C, WKYMVm
Neutrophils are generated from hematopoietic stem cells and their subsequent intermediate progenitors such as common myeloid progenitors (CMP) and granulocyte-macrophage progenitors (GMP) $(4,5)$. Such de novo neutrophil generation has been observed in steady-state granulopoiesis in local bacterial infection and in emergency granulopoiesis for systemic bacterial infection (6). Unlike steady-state granulopoiesis, emergency granulopoiesis rapidly generates an increased number of neutrophils in response to an emergency alert signal following systemic bacterial infection (6). Therefore, it is of great interest to reveal molecular mechanisms involved in the regulation of emergency granulopoiesis.

WKYMVm is a synthetic peptide that stimulates innate immune cells such as neutrophils, monocytes, and macrophages (7-10). WKYMVm stimulates chemotactic migration and ROS generation in neutrophils and monocytes (7-9). On cell surface receptor for this peptide, it binds to formyl peptide receptor (FPR) family members including FPR1 and FPR2 (11, 12). Previously, we have demonstrated that WKYMVm administration has a therapeutic effect against sepsis mainly through FPR2 (13). Regarding the mechanism involved in the antiseptic activity of WKYMVm, the peptide can stimulate IFN- $\gamma$ production and suppress proinflammatory cytokine production such as the production of TNF- $\alpha$ and IL-1 $\beta$ (13). However, the functional role of WKYMVm and its cognitive receptor(s) in emergency granulopoiesis remain unclear.

Phospholipase C (PLC) is an important cell signaling enzyme that can be activated by stimulation of cell surface receptors such as G-protein coupled receptors or receptor tyrosine kinase (14). PLC hydrolyzes membrane phospholipid phosphoinositide 4,5-bisophosphate to inositol 1,4,5-trisphosphate and diacylglycerol. The generated inositol 1,4,5-trisphosphate can subsequently induce cytosolic calcium increase while diacylglycerol can activate protein kinase C (14). Several different isoforms of PLC have been reported, including PLC $\beta$ $(\beta 1-\beta 4), \operatorname{PLC} \gamma(\gamma 1, \gamma 2), \operatorname{PLC} \delta(\delta 1-\delta 4), \operatorname{PLC} \varepsilon 1, \operatorname{PLC} \eta(\eta 1, \eta 2)$, and PLC 1 . These PLC isoforms can mediate diverse cellular responses including cell proliferation and cell migration (15).

ISSN: 1976-670X (electronic edition)

Copyright (c) 2018 by the The Korean Society for Biochemistry and Molecular Biology

(c) This is an open-access article distributed under the terms of the Creative Commons Attribution Non-Commercial License (http://creativecommons.org/licenses/by-nc/4.0) which permits unrestricted non-commercial use, distribution, and reproduction in any medium, provided the original work is properly cited. 
Some PLC isoforms are also associated with tumorigenesis and cancer metastasis $(16,17)$. However, the functional role of $\mathrm{PLC}$ in the regulation of emergency granulopoiesis has not been reported yet.

The objective of this study was to determine whether WKYMVm administration in cecal ligation puncture (CLP) mice could stimulate emergency granulopoiesis and characterize the functional role of FPR2 and PLC in WKYMVm peptide-stimulated emergency granulopoiesis.

\section{RESULTS}

WKYMVm increases neutrophil number in peritoneal fluid and peripheral blood in sepsis

Neutrophils $\left(\mathrm{CD} 11 \mathrm{~b}^{+} \mathrm{Ly}_{6 \mathrm{G}}{ }^{+}\right)$play essential roles in defense against polymicrobial sepsis by killing invading bacteria (18). Since WKYMVm administration elicited strong bacterial killing activity in CLP sepsis mice (13), we investigated whether this peptide could affect neutrophil recruitment into the event area. CLP surgery caused an increase of neutrophils in peritoneal fluid. Administration of WKYMVm in CLP mice further increased the number of neutrophils in peritoneal fluid (Fig. 1A). Maximal activity of neutrophil increase in the peritoneal fluid was observed after administration of WKYMVm at 8 $\mathrm{mg} / \mathrm{kg}$ (Fig. 1A). We also found that WKYMVm increased neutrophil number in peripheral blood in CLP mice in a dose-dependent manner, showing maximal activity at $8 \mathrm{mg} / \mathrm{kg}$ (Fig. 1B).

\section{WKYMVm stimulates emergency granulopoiesis}

Since we found that WKYMVm administration elicited an increase of neutrophil count in peripheral blood in the CLP sepsis model (Fig. 1B), we investigated whether WKYMVm could affect neutrophil number in CLP mice. Neutrophil
A

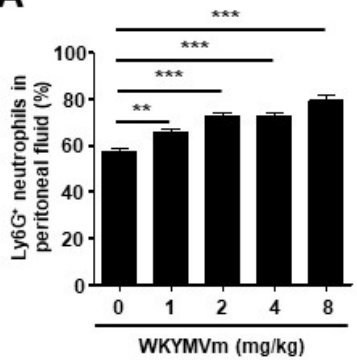

B

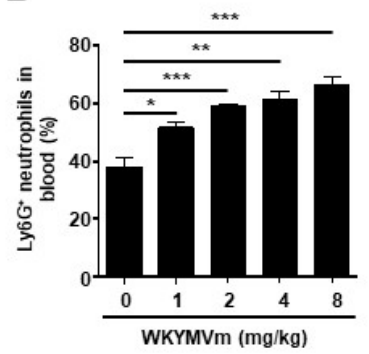

Fig. 1. WKYMVm stimulates increase of neutrophils in CLP sepsis model. (A, B) Vehicle $(0.8 \%$ DMSO in PBS) or WKYMVm $(0,1$, 2, 4, $8 \mathrm{mg} / \mathrm{kg}$ ) was i.p. administered at $2 \mathrm{~h}$ after CLP. (A) Peritoneal lavage fluid neutrophils and (B) peripheral blood neutrophils collected $24 \mathrm{~h}$ after CLP were measured by flow cytometry after staining cells with an anti-CD11b antibody and an anti-Ly6G antibody. Data are expressed as means \pm SEM $(n=4)$ (A, B). ${ }^{* P}<0.05, * * P<0.01, * * * P<0.001$. numbers in bone marrow (BM) were compared between vehicle- and WKYMVm-administrated CLP mice. WKYMVm administration increased neutrophil number in the $\mathrm{BM}$ in the CLP sepsis model (Fig. 2A). We then examined effects of WKYMVm administration on the generation of GMP, a neutrophil progenitor. After gating $\mathrm{Lin}^{-}$populations, LK ( in $^{-} \mathrm{C}-\mathrm{Kit}^{+} \mathrm{Sca}-{ }^{-}$) cell population was further analyzed by staining with anti-CD34 and anti-FcyRII/III (CD16/32). GMP was identified as $\mathrm{CD} 34^{+} \mathrm{CD} 16 / 32^{+}$as shown in Fig. $2 \mathrm{~B}$. megakaryocyte erythrocyte progenitors (MEP) and CMP were identified as $\mathrm{CD}_{4} 4^{-} \mathrm{CD} 16 / 32^{-}$and $\mathrm{CD} 34^{+} \mathrm{CD} 16 / 32^{-}$, respectively (Fig. 2B). WKYMVm administration significantly increased the number of GMP in CLP sepsis mice (Fig. 2B, 2C). We also examined whether WKYMVm administration increased GMP population in non-CLP mice. Administration of WKYMVm at different doses in non-CLP mice did not significantly affect GMP population (Fig. 2D). These results suggest that WKYMVm can specifically stimulate emergency granulopoiesis by increasing GMP population.

\section{WKYMVm-stimulated emergency granulopoiesis is mediated by FPR2}

WKYMVm administration increased neutrophil number in the CLP sepsis model (Fig. 1). The stimulatory effect of WKYMVm on the generation of neutrophils was mediated by enhancing

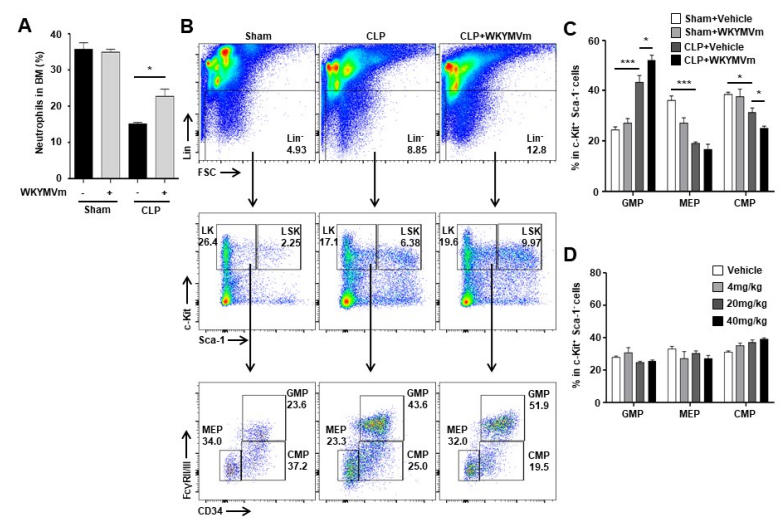

Fig, 2. WKYMVm stimulates emergency granulopoiesis in CLP septic mice. (A) Vehicle $(0.8 \%$ DMSO in PBS) or WKYMVm (4 $\mathrm{mg} / \mathrm{kg}$ ) was i.p. administered at 2 and $14 \mathrm{~h}$ after CLP. BM cells were collected at $24 \mathrm{~h}$ after sham or CLP surgery. The percentage of neutrophils in BM cells was measured using flow cytometry. (B) Flow cytometry-based granulopoiesis analysis of BM cells isolated at $24 \mathrm{~h}$ after CLP. (C) Flow cytometric analysis of lin $^{-}{\mathrm{C}-\mathrm{Kit}^{+}}^{+} \mathrm{Sca}-{ }^{-}$BM cells from C57BL/6 mice $24 \mathrm{~h}$ after CLP. (D) Vehicle $(0.8 \%$ DMSO in PBS) or WKYMVm $(4,20,40$ $\mathrm{mg} / \mathrm{kg}$ ) was i.p. administered at 2 and $14 \mathrm{~h}$ after sham operation. Flow cytometric analysis of $\operatorname{lin}^{-} \mathrm{C}-\mathrm{Kit}^{+} \mathrm{Sca}-1^{-}$BM cells from $\mathrm{C} 57 \mathrm{BL} / 6$ mice was done at $24 \mathrm{~h}$ after sham surgery. Numbers indicate percentages of CMP, GMP, and MEP within lin $^{-}{\mathrm{C}-\mathrm{Kit}^{+}}^{+} \mathrm{Sca}-1^{-}$BM cell population (C, D). Data are expressed as means $\pm \operatorname{SEM}(\mathrm{n}=5)$. ${ }^{*} \mathrm{P}<0.05, * * * \mathrm{P}<0.001$. 
the generation of neutrophil precursors, GMP (Fig. 2B, 2C). It has been previously reported that WKYMVm can bind to FPR1 and FPR2 in mice $(11,12)$. In this study, we examined whether LK cells, progenitors of neutrophils, could express FPR 1 and FPR2 using RT-PCR analysis. As shown in Fig. 3A, LK cells expressed both FPR1 and FPR2. Since WKYMVm enhanced emergency granulopoiesis in CLP mice, we investigated whether FPR expression in LK cells was changed in septic mice. FPR2 expression was not affected by CLP operation. However, FPR1 expression in LK cells was slightly decreased (Fig. 3B). We then investigated which receptor was involved in this peptide-stimulated granulopoiesis using an FPR1 antagonist (cyclosporine $\mathrm{H}, \mathrm{CsH}$ ) or an FPR2 antagonist (WRWWWW, WRW4) (19). As shown in Fig. 3C, WKYMVmstimulated emergency granulopoiesis was significantly inhibited by WRW4, but not by $\mathrm{CsH}$. These results suggest that FPR2 can mediate WKYMVm-stimulated emergency granulopoiesis in CLP sepsis mice. WKYMVm-elicited emergency granulopoiesis was only observed from littermate wild-type (WT) mice, but not from FPR2 knockout (KO) mice (Fig. 3D). These results strongly support the notion that FPR2 plays an essential role in WKYMVm-elicited emergency granulopoiesis.
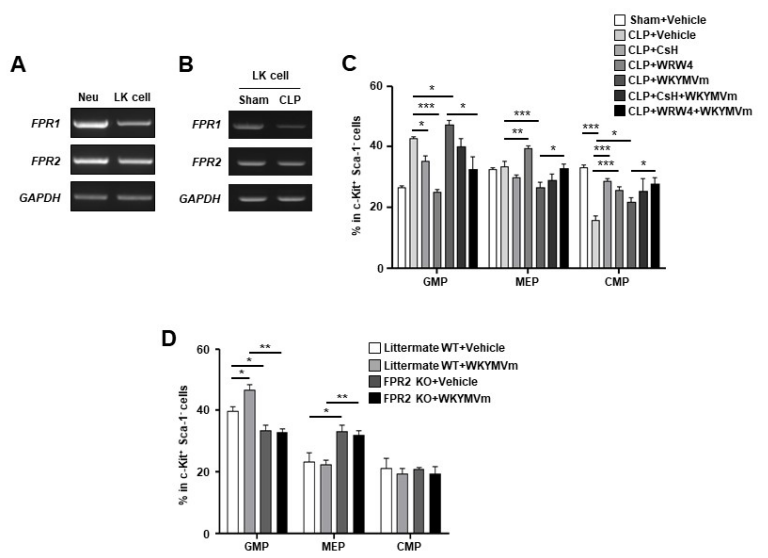

Fig. 3. WKYMVm-induced emergency granulopoiesis is mediated by FPR2. (A) Expression levels of FPR1 and FPR2 from BM neutrophils or BM LK cells were analyzed by RT-PCR. (B) Expression levels of FPR1 and FPR2 from BM LK cells of sham or CLP mice were analyzed by RT-PCR. (C) $\mathrm{CsH}(4 \mathrm{mg} / \mathrm{kg})$ or WRW4 (4 mg/kg) was i.p. injected at $2 \mathrm{~h}$ before CLP. Vehicle $(0.8 \%$ DMSO in PBS) or WKYMVm $(4 \mathrm{mg} / \mathrm{kg})$ was administered at 2 and $14 \mathrm{~h}$ after CLP. (D) Vehicle (0.8\% DMSO in PBS) or WKYMVm (4 mg/kg) was i.p. administered at 2 and $14 \mathrm{~h}$ after CLP in littermate WT or FPR2 KO mice. BM cells were collected at $24 \mathrm{~h}$ after CLP surgery. Numbers indicate percentages of CMP, GMP, and MEP within lin ${ }^{-} \mathrm{C}-\mathrm{Kit}^{+} \mathrm{Sca}-1^{-} \mathrm{BM}$ cell population. Data are expressed as means \pm SEM $(n=5)$. ${ }^{*} P<0.05, * * P<$ $0.01, * * * \mathrm{P}<0.001$. Data are representatives of three independent experiments $(\mathrm{A})$.

\section{PLC plays a role in WKYMVm-stimulated emergency granulopoiesis}

WKYMVm is known to stimulate PLC activity, resulting in strong increase of calcium signaling $(7,9)$. Thus, we investigated the role of PLC in WKYMVm-induced neutrophil recruitment and neutrophil number increase in CLP mice. We found that WKYMVm-induced further increase of neutrophil recruitment into peritoneal fluid was blocked by U-73122 (a PLC inhibitor), but not by U-73343 (inactive control of U-73122) (Fig. 4A). WKYMVm-induced neutrophil number increase in peripheral blood in CLP mice was also blocked by U-73122 (Fig. 4B).

We also examined the effect of PLC activity on WKYMVminduced emergency granulopoiesis using a PLC inhibitor. Administration of a PLC inhibitor U-73122 prior to WKYMVm administration inhibited WKYMVm-induced increase in the percentage of c-kit ${ }^{+}$sca- ${ }^{-}$GMP (Fig. 4C). WKYMVm-induced increase in the absolute number of GMP was also significantly blocked by U-73122, but not by U-73343 (Fig. 4D). These results suggest that WKYMVm-stimulated emergency granulopoiesis is mediated by PLC activity.

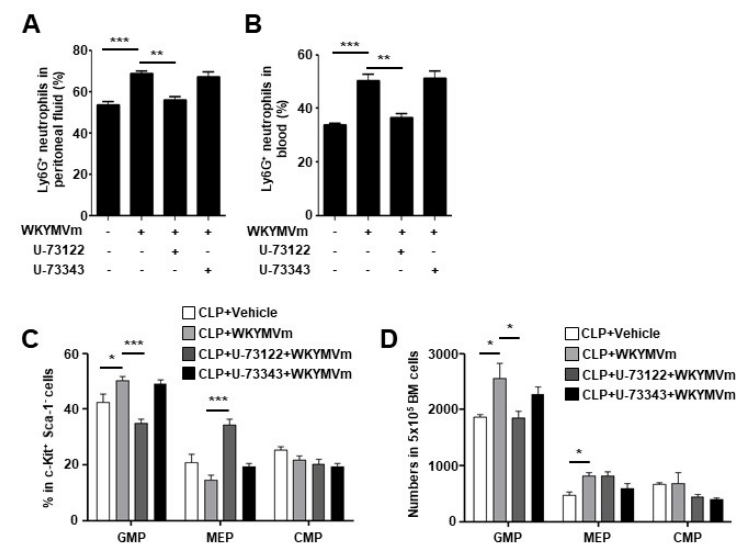

Fig. 4. WKYMVm-induced emergency granulopoiesis is mediated by PLC activity. (A-D) U-73122 (1.5 mg/kg) or U-73343 (1.5 $\mathrm{mg} / \mathrm{kg}$ ) was i.p. injected at $2 \mathrm{~h}$ before CLP. Vehicle $(0.8 \%$ DMSO in PBS) or WKYMVm (4 mg/kg) was i.p. administered at $2 \mathrm{~h}$ or at $2 \mathrm{~h}$ and $14 \mathrm{~h}$ after CLP. (A) Peritoneal lavage fluid neutrophils and (B) peripheral blood neutrophils collected $6 \mathrm{~h}$ after CLP, CLP + WKYMVm, CLP + U-73122 + WKYMVm, or CLP + U-73343 + WKYMVm administration were measured by flow cytometry after staining cells with an anti-CD11b antibody and an anti-Ly6C antibody. (C, D) BM cells were collected at $24 \mathrm{~h}$ after CLP surgery. The percentage of neutrophils in BM cells was measured by flow cytometry. Numbers indicate percentages of CMP, GMP,

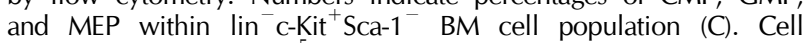
numbers (per $5 \times 10^{5} \mathrm{BM}$ cells) of myeloid progenitors are shown (D). Data are expressed as means \pm SEM $(n=5)$ (A-D). $* P<0.05$, **P $<0.01$, ***P $<0.001$. 


\section{DISCUSSION}

Although emergency granulopoiesis is important for rapid generation of neutrophils in the presence of a systemic bacterial infection $(6,20)$, the regulatory mechanism has not been characterized yet. Which cell surface receptor is involved in the positive regulation of emergency granulopoiesis remains unclear. In this study, we demonstrate that WKYMVm known to bind to FPR family members can stimulate emergency granulopoiesis. As neutrophil progenitors, LK cells expressed FPR1 and FPR2 (Fig. 3A). WKYMVmstimulated emergency granulopoiesis was specifically blocked by WRW4, but not by $\mathrm{CsH}$ (Fig. 3C). Furthermore, WKYMVminduced emergency granulopoiesis was significantly blocked by FPR2 deficiency (Fig. 3D). Since WKYMVm is a surrogate agonist for FPR1 and FPR2 in mouse phagocytic cells such as neutrophils and monocytes, our results suggest that activation of FPR family members (especially FPR2) can induce emergency granulopoiesis. To the best of our knowledge, this is the first report to demonstrate functional regulation of emergency granulopoiesis by a chemoattractant G-protein coupled receptor.

FPR recognizes bacterial formyl peptides $(21,22)$. Systemic bacterial infection may induce the activation of FPR in BM neutrophil precursors such as CMP and GMP. Since FPR can detect bacteria-derived $\mathrm{N}$-formylated peptide (21), recognition of this bacteria-derived entity may initiate an alarm signal to induce emergency granulopoiesis. In this study, we found that the addition of FPR2 antagonist (WRW4) to CLP model could decrease GMP fraction below the level without WKYMVm addition (Fig. 3C). Similarly, FPR2-deficient mice showed decreased GMP fraction without peptide administration (Fig. 3D). These results suggest that FPR2 plays a critical role in granulopoiesis in the absence of exogenous stimuli. However, activation of FPR is insufficient to induce emergency granulopoiesis without CLP operation because WKYMVm administration alone does not induce emergency granulopoiesis (Fig. 2D). WKYMVm-induced emergency granulopoiesis requires CLP. These results suggest that CLP operation-induced septic conditions may contribute to the initiation of emergency granulopoiesis which is mediated by FPR2, at least partly, and that WKYMVm can augment this CLP-initiated emergency granulopoiesis.

The role of PLC activity in emergency granulopoiesis has not been reported yet. In this study, we demonstrated that activation of PLC by WKYMVm stimulation could elicit an increase in the number of neutrophils by upregulating emergency granulopoiesis. To the best of our knowledge, this is the first report to demonstrate the functional role of PLC in GMP generation and emergency granulopoiesis. Since previous studies have demonstrated that stimulation of G-protein coupled receptors can induce the activation of PLC $\beta$ isoforms $(23,24)$, which specific isoform of PLC is involved in
WKYMVm-induced emergency granulopoiesis needs to be investigated in the future.

In conclusion, we demonstrate that activation of FPR2 by its surrogate agonist, WKYMVm, can augment emergency granulopoiesis in a CLP model. We also revealed the functional role of FPR2 and PLC in WKYMVm peptidestimulated emergency granulopoiesis. Our results provide a novel insight into the important function of FPR2 and PLC in neutrophil generation.

\section{MATERIALS AND METHODS}

\section{Materials}

WKYMVm and WRW4 were synthesized by Anygen (Gwangju, Korea). PLC inhibitor U-73122 and its inactive analogue U-73343 were obtained from Calbiochem (San Diego, CA, USA). CsH was purchased from Enzo life sciences (Farmingdale, NY, USA).

\section{Mice and CLP experimental sepsis model}

Male WT 8-week-old C57BL/6 mice were purchased from Orient Bio (Seongnam, Korea). FPR2 KO mice were generated as described previously (25). All experiments involving animals adhered to relevant guidelines and received approval from the Institutional Review Committee for Animal Care and Use at Sungkyunkwan University (Suwon, Korea). The experimental CLP sepsis model was induced as described previously $(26,27)$. After midline laparotomy, we exposed the cecum, ligated below the ileocecal valve without causing intestinal obstruction, and then punctured the cecum twice with a 22-gauge needle. Sham mice were subjected to the same procedure without puncture of the cecum.

\section{Emergency granulopoiesis and neutrophil population analysis}

Emergency granulopoiesis was analyzed according to a previous report (20). Briefly, WT, littermate WT, and FPR2 KO mice used for analysis were males from 7 to 9 weeks old. Cells were surface-stained in PEB buffer (PBS supplemented with and $2 \mathrm{mM}$ EDTA and $0.5 \% \mathrm{BSA}$ ) for $30-45 \mathrm{~min}$ on ice. Antibodies used for flow cytometry included the following: Percp-Cy5.5-conjugated lineage markers specific for CD3e (Clone: 145-2C110), CD4 (Clone: RM4-5), CD8a (Clone: 53-6.7), CD11b (Clone: M1/70), B220 (Clone: RA3-6B2), GR-1 (Clone: RB6-8C5), and Ter119 (Clone: TER119) from Thermo Fisher scientific (Waltham, MA, USA) or BioLegend (San Diego, CA, USA). Other antibodies included those against APC- or PE-conjugated Sca-1 (Clone: D7), c-kit (Clone: 2B8), FITC- or PE-Cy7-conjugated CD16/32 (Clone: 93), and CD34 (Clone: RAM34). Neutrophils were gated by $\mathrm{CD}_{11 \mathrm{~b}^{+}}$and ${\text { Ly } 6 G^{+}}^{+}$(Clone: 1A8). Unstained cells were used as negative controls to establish flow cytometer voltage settings and single-color positive controls were used to adjust the compensation. Flow cytometric data were acquired using 
FACScantoll and analyzed with Flowjo software (Tree Star Inc. Ashand, OR, USA).

\section{BM neutrophil and LK cell sorting}

BM cells were isolated from femurs using HBSS-EDTA solution. The cell suspension was centrifuged at $460 \mathrm{~g}$ for 5 min. Resuspended cells were carefully loaded onto $40 \%$, 47\%, and $60 \%$ Percoll gradient and centrifuged at 2,900 g for 30 min without braking. Cells were isolated on the $60 \% / 47 \%$ interface layer and red blood cells were removed by hypotonic lysis. Isolated cells were over 90\% Ly6G-positive by flow cytometry. For LK cell sorting, BM cells were stained with Percp-Cy5.5-conjugated lineage-specific markers, PE-conjugated c-kit (Clone: 2B8), and APC conjugated Sca-1 (Clone: D7). Cells were then sorted through $\mathrm{Lin}^{-} \mathrm{C}-\mathrm{kit}^{+} \mathrm{Sca}-{ }^{-}$for LK cells using FACS Arialll equipped with FACSDiva software (BD Bioscience).

\section{Reverse transcription-PCR (RT-PCR) analysis}

Primer used for RT-PCR included the following: FPR 1 forward, 5'-ACAGCCTGTACTTTCGAC-3', FPR 1 reverse, 5'-CTGGAAG TTAGAGCCCGTTC-3'; FPR2 forward, 5'-GTCAAGATCAAC AGAAGAAACC-3', FPR2 reverse, 5'-GGGCTCTCTCAAGAC TATAAGG-3'; GAPDH forward, 5'-TCCACCACCCTGTTGCT GTA-3', GAPDH reverse, 5'-AATGTGTCCGTCGTGGATCT-3'. RT-PCR was performed with 34 PCR cycles of $94^{\circ} \mathrm{C}$ (denaturation, $30 \mathrm{~s}$ ), $62^{\circ} \mathrm{C}$ (annealing, $45 \mathrm{~s}$ ), and $72^{\circ} \mathrm{C}$ (extension, $1 \mathrm{~min}$ ). PCR products were electrophoresed on $1 \%$ or $2 \%$ agarose gel and visualized after ethidium bromide staining.

\section{Statistical analysis}

All results were evaluated using GraphPad prism software. Statistical analysis was done using one-way analysis of variance (ANOVA) followed by Student's $t$-test. All results are expressed as mean \pm SEM. A P value $<0.05$ was considered statistically significant.

\section{ACKNOWLEDGEMENTS}

This work was supported by Basic Science Research Program through the National Research Foundation of Korea (NRF) funded by the Ministry of Science, ICT and Future Planning (NRF-2015R1A2A1A10054567, NRF-2018R1A2B3003868).

\section{CONFLICTS OF INTEREST}

The authors have no conflicting interests.

\section{REFERENCES}

1. Smith JA (1994) Neutrophils, host defense, and inflammation: a double-edged sword. J Leukoc Biol 56, 672-686

2. Kruger P, Saffarzadeh M, Weber ANR et al (2015)
Neutrophils: Between host defence, immune modulation, and tissue injury. PLoS Pathog 11, e1004651

3. DeCoursey TE (2016) The intimate and controversial relationship between voltage-gated proton channels and the phagocyte NADPH oxidase. Immunol Rev 273, 194-218

4. Iwasaki H, Akashi K (2007) Myeloid lineage commitment from the hematopoietic stem cell. Immunity 22, 726-740

5. Seita J, Weissman IL (2010) Hematopoietic stem cell: self-renewal versus differentiation. Wiley Interdiscip Rev Syst Biol Med 2, 640-653

6. Manz MG, Boettcher S (2014) Emergency granulopoiesis. Nat Rev Immunol 14, 302-314

7. Seo JK, Choi SY, Kim Y et al (1997) A peptide with unique receptor specificity: stimulation of phosphoinositide hydrolysis and induction of superoxide generation in human neutrophils. J Immunol 158, 1895-1901

8. Bae YS, Ju SA, Kim JY et al (1999) Trp-Lys-TyrMet-Val-D-Met stimulates superoxide generation and killing of Staphylococcus aureus via phospholipase D activation in human monocytes. J Leukoc Biol 65, 241-248

9. Bae YS, Kim Y, Kim JH et al (2000) Independent functioning of cytosolic phospholipase A2 and phospholipase D1 in Trp-Lys-Tyr-Met-Val-D-Met-induced superoxide generation in human monocytes. J Immunol 164, 4089-4096

10. Schepetkin IA, Kirpotina LN, Tian J et al (2008) Identification of novel formyl peptide receptor-like 1 agonists that induce macrophage tumor necrosis factor $\alpha$ Production. Mol Pharmacol 74, 392-402

11. Le Y, Gong W, Li B et al (1999) Utilization of two seven-transmembrane, G protein-coupled receptors, formyl peptide receptor-like 1 and formyl peptide receptor, by the synthetic hexapeptide WKYMVm for human phagocyte activation. J Immunol 163, 6777-6784

12. Bae YS, Song JY, Kim Y et al (2003) Differential activation of formyl peptide receptor signaling by peptide ligands. Mol Pharmacol 64, 841-847

13. Kim SD, Kim YK, Lee HY et al (2010) The agonists of formyl peptide receptors prevent development of severe sepsis after microbial infection. J Immunol 185, 4302-4310

14. Noh DY, Shin SH, Rhee SG (1995) Phosphoinositidespecific phospholipase $\mathrm{C}$ and mitogenic signaling. Biochim Biophys Acta 1242, 99-113

15. Suh PG, Park JI, Manzoli L et al (2008) Multiple roles of phosphoinositide-specific phospholipase $\mathrm{C}$ isozymes. BMB Rep 41, 415-434

16. Lattanzio R, Piantelli M, Falasca M (2013) Role of phospholipase $\mathrm{C}$ in cell invasion and metastasis. Adv Biol Regul 53, 309-318

17. Cheng M, Bhujwalla ZM, Glunde K (2016) Targeting phospholipid Metabolism in cancer. Front Oncol 6, 266

18. Sônego F, Castanheira FV, Ferreira RG et al (2016) Paradoxical roles of the neutrophil in sepsis: protective and deleterious. Front Immunol 7, 155

19. Bae YS, Lee HY, Jo EJ et al (2004) Identification of peptides that antagonize formyl peptide receptor-like 1-mediated signaling. J Immunol 173, 607-614

20. Kwak HJ, Liu P, Bajrami B et al (2015) Myeloid 
cell-derived reactive oxygen species externally regulate the proliferation of myeloid progenitors in emergency granulopoiesis. Immunity 42, 159-171

21. Ye RD, Boulay F, Wang JM et al (2009) International union of basic and clinical pharmacology. LXXIII. nomenclature for the formyl peptide receptor (FPR) family. Pharmacol Rev 61, 119-161

22. Lee HY, Lee M, Bae YS (2017) Formyl peptide receptors in cellular differentiation and inflammatory diseases. J Cell Biochem 6, 1300-1307

23. Rhee SG (2001) Regulation of phosphoinositide-specific phospholipase C. Annu Rev Biochem 70, 281-312

24. Zhang S, Coso OA, Collins R, Gutkind JS, Simonds WF (1996) A C-terminal mutant of the G protein beta subunit deficient in the activation of phospholipase C-beta. J Biol Chem 271, 20208-20212

25. Park MY, Kim HS et al (2017) FAM19A5, a brain-specific chemokine, inhibits RANKL-induced osteoclast formation through Formyl peptide receptor 2. Sci Rep 7, 15575

26. Lee SK, Kim SD, Kook MS et al (2015) Phospholipase D2 drives mortality in sepsis by inhibiting neutrophil extracellular trap formation and down-regulating CXCR2. J Exp Med 212, 1381-1390

27. Kim SD, Lee HY, Shim JW et al (2011) Activation of CXCR2 by extracellular matrix degradation product acetylated Pro-Gly-Pro has therapeutic effects against sepsis. Am J Respir Crit Care Med 184, 243-251 